\title{
Galaxy Clustering in the Southern Hemisphere
}

\section{C.A. Collins}

Astrophysics Group, School of Chemical and Physical Sciences, Liverpool John Moores University, Byrom Street, Liverpool L3 $3 A F$

\begin{abstract}
In this paper some of the major results from the COSMOS and APM digitised galaxy surveys are presented. The main motivation behind these catalogues was to study large-scale structure in the universe. We begin by outlining the importance of such studies to cosmology and discussing the early results from the visually compiled galaxy catalogues. The impact of the digitised catalogues is demonstrated by focussing on three key areas of research; the galaxy-galaxy two-point angular correlation function, the cluster-cluster spatial correlation function, and galaxy number counts.
\end{abstract}

\section{Introduction}

One of the key problems in cosmology is to explain the origin of galaxies, clusters of galaxies and large-scale structure in the universe. On sufficiently large scales, gravity has not had time to significantly change the relative positions of galaxies and their distribution becomes an accurate fossil record of the fluctuations in the universe. An estimate of the typical scale-size above which gravity has not played an important role can be found from a consideration of peculiar velocities. Since the typical peculiar velocity $\left(\mathrm{V}_{\mathrm{p}}\right)$ of a field galaxy, or cluster of galaxies, is $\simeq 500 \mathrm{~km} \mathrm{sec}^{-1}$ (Aaronson et al. 1986, Lucey \& Carter 1988), galaxies will not move more than a distance $\sim \mathrm{H}_{0}^{-1} \mathrm{~V}_{\mathrm{p}} \sim 5 h^{-1} \mathrm{Mpc}$, over the lifetime of the universe, where $\mathrm{H}_{0}$ is the Hubble constant and $h=\mathrm{H}_{0} / 100 \mathrm{~km} \mathrm{~s}^{-1} \mathrm{Mpc}^{-1}$. On larger scales than this, the distribution of structure should still accurately reflect the initial power spectrum of density fluctuations.

The most widely used statistic to characterize the amplitude of galaxy clustering is the two-point spatial correlation function $\xi(r)$, defined as

$$
d P(r)=n\left(r_{1}\right) n\left(r_{2}\right)[1+\xi(r)] d V_{1} d V_{2},
$$

where $d P(r)$ denotes the joint probability of finding objects in each of the volume elements $d V_{1}$ and $d V_{2}$, separated by distance $r$, and $n\left(r_{1}\right), n\left(r_{2}\right)$ are the number densities of such objects at positions 1,2 respectively. A corresponding definition applies to $w(\theta)$, the angular two-point correlation function. Both $\xi(r)$ and $w(\theta)$ are directly related to the spectrum of density fluctuations by means of a Fourier transform. In addition, on scales $\geq 5 h^{-1} \mathrm{Mpc}$, the overdensities are less than unity and linear theory can be used to describe the evolution of the density field (see Efstathiou 1990). Therefore, using some prescription for how the light we 
observe traces the underlying mass, observations on large scales can be used to directly constrain the initial power spectrum.

\section{The Lick Survey and Abell Catalogue}

Two of the largest and most widely used galaxy catalogues are the Lick catalogue of galaxies (Shane \& Wirtanen 1967), and the Abell catalogue of rich galaxy clusters (Abell 1958, Abell et al. 1989). The Lick catalogue was compiled from visual scans of the Lick astrographic survey, over a period of seven years. In total, $\simeq 8 \times 10^{5}$ galaxies were counted in $10 \times 10 \mathrm{arcmin}^{2}$ cells, on 1246 plates $\left(\right.$ each $6^{\circ} \times 6^{\circ}$ ), covering an area $\simeq 3000 \mathrm{deg}^{2}$. The Abell catalogue contains some 4000 galaxy clusters covering both hemispheres. The initial catalogue (Abell 1958) was limited to the declination zone available to the Palomar Sky Survey (north of $-27^{\circ}$ ). Abell visually estimated cluster centroids, cluster richness, compactness and the distance to each cluster. More recently, the cluster catalogue was completed in the southern hemisphere by H. Corwin and R. Olowin, using the UK Schmidt IIIa-J survey plates and following, as closely as possible, the selection procedure used by Abell (Abell et al. 1989).

Results from the statistical analysis of these catalogues proved extremely influential and the conclusions drawn went a long way to directing the course of theoretical cosmology for the next twenty years. Some of the more famous results are:

- Galaxies show a power-law clustering $w(\theta)=\theta^{(1-\gamma)}$ on scales $\theta \leq 2.5^{\circ}$, with $\gamma=1.8$. In addition, there is evidence of a break from a powerlaw form on an angular scale $\theta \simeq 2.5^{\circ}$, corresponding to a distance of $\simeq 9 h^{-1} \mathrm{Mpc}$ (Groth \& Peebles 1977).

- Rich clusters of galaxies have a spatial correlation function of the form $\xi_{c c}(r)=\left(r / r_{0}\right)^{-1.8}$, with $r_{0}=20-25 h^{-1} \mathrm{Mpc}$ (Bahcall \& Soneira 1983; Postman et al. 1986, 1992).

- The distribution of clusters is anisotropic, with more cluster pairs along the radial or redshift direction than in the transverse direction. This result implies that either superclusters are preferentially aligned along the lineof-sight or that clusters have peculiar velocities $\simeq 2000 \mathrm{~km} \mathrm{sec}^{-1}$ (Bahcall et al. 1986, Jing et al. 1992).

Despite a vigorous defence of these clustering results by some authors (Groth \& Peebles 1986a, 1986b; Bahcall \& West 1992), it is most likely that they are either wrong or at least in need of serious revision. The major criticisms centre on the subjective nature of the catalogues and the systematic errors introduced by the visual scanning of the plates (Geller et al. 1984; Sutherland (1988); Efstathiou et al. 1992a). In any case, there is little disagreement regarding the potential improvements using digitised data and I will indicate in the next few sections in what way these results have been overturned by the new surveys. 


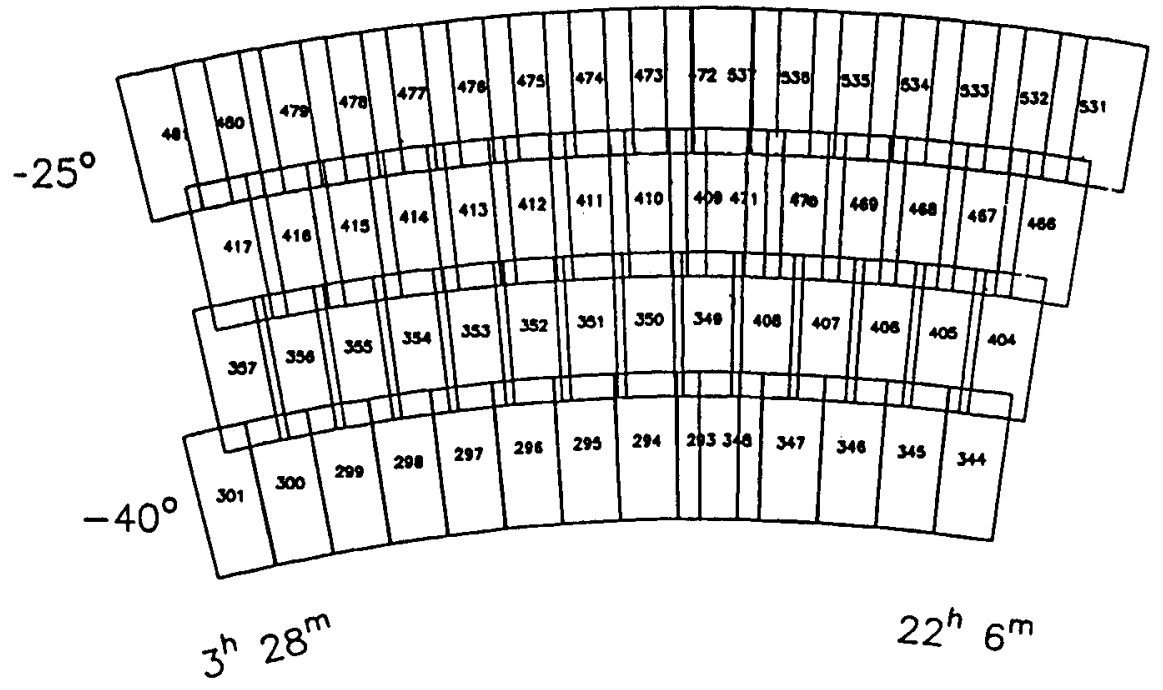

Figure 1. The IIIa-J Schmidt fields which constitute the EDSGC

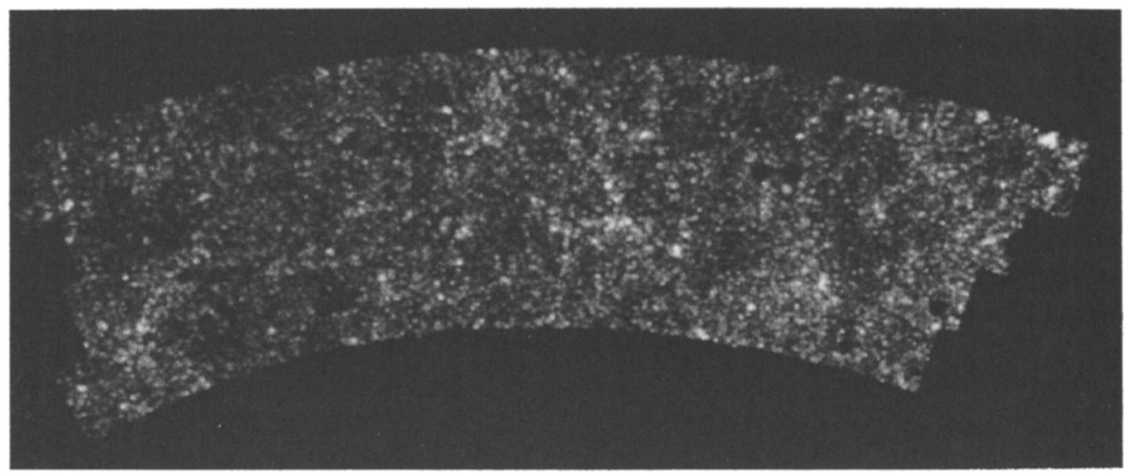

Figure 2. The EDSGC as an equal area projection, with the galaxies binned in $10 \times 10$ arcmin bins at a depth of $b_{j}=18.5$ 


\section{Large Digitised Surveys}

Several years ago two projects were started in the UK, with the aim of scanning large numbers of the UK Schmidt IIIa-J survey in order to construct objective and homogeneous galaxy catalogues. These are the Edinburgh Durham Southern Galaxy Catalogue (Heydon-Dumbleton 1989), and the APM Galaxy Catalogue (Maddox et al. 1990).

The Edinburgh Durham Southern Galaxy Catalogue (EDSGC) consists of 60 IIIa-J plates, scanned by the COSMOS measuring machine in Edinburgh (see Figure 1 and Figure 2). The original APM galaxy catalogue consists of 185 plates from the same material, scanned by the APM measuring facility in Cambridge. Star galaxy classification in the EDSGC is $\leq 10 \%$ and the completeness is $>$ $95 \%$. In addition, there is a $3 \% \mathrm{rms}$ residual variation in the number of objects classed as galaxies from plate-to-plate (Heydon Dumbleton 1989). The EDSGC is calibrated using CCD galaxy sequences on almost half the fields in the survey. Fields without sequences are calibrated using the overlaps between the plates (Collins et al. 1992). The residuals in the plate overlaps indicate an rms 0.05 mag. The APM group calibrate by relying on the plate overlaps to remove the plate-to-plate variations, leaving their CCD sequences to set the zero-point of the photometric system for the entire survey. This procedure naturally produces a small rms in the overlaps between plates $(2 \%-3 \%)$. However, Fong et al. (1992) point out that this method may admit small residual plate-to-plate zero-point errors to be propagated over large areas of the survey. In any event, it is certainly the case that both surveys could be improved with the addition of further CCD calibration sequences. However, the response of the photographic emulsion is non-linear and the fundamental problem is to correct for this in a way which does not leave residual errors. Consequently, a great number of sequences are probably required to make a significant impact on the photometric accuracy of the EDSGC and APM surveys (Fong et al. 1992). Finally, the EDSGC is corrected for galactic extinction using the HI maps of Stark et al. (1992). Using typical values for the reddening ratio and dust to gas ratio from the literature (Mathis 1990), the Stark data imply extinction levels corresponding to $A_{B} \simeq 0.1$ at the SGP and so cannot be ignored (Nichol \& Collins 1992).

\section{The Galaxy Angular Correlation Function}

The digitised surveys can constrain the power on scales $\geq 10 h^{-1} \mathrm{Mpc}$ in two dimensions, better than current redshift surveys, simply because they contain an overwhelming number of galaxies, $\sim 10^{6}$. In addition, $w(\theta)$ is unaffected by peculiar velocities of galaxies, whereas they have an important affect on the three dimensional correlation function. In Figure 3 the EDSGC $w(\theta)$ is shown at four different magnitude limits along with those of the Lick survey (Groth \& Peebles 1977), the APM survey (Maddox et al. 1990), and the results from the POSS II digitised survey of Picard (1991), all scaled to the depth of the Lick survey. The digitised survey results illustrate several new features. First, the correlation function has a slow "role-off" and the sharp break feature at $2.5^{\circ}$ observed by Groth \& Peebles (1977) in the Lick data is not so prominent. Secondly, the correlation function remains positive out to an angular scale of 


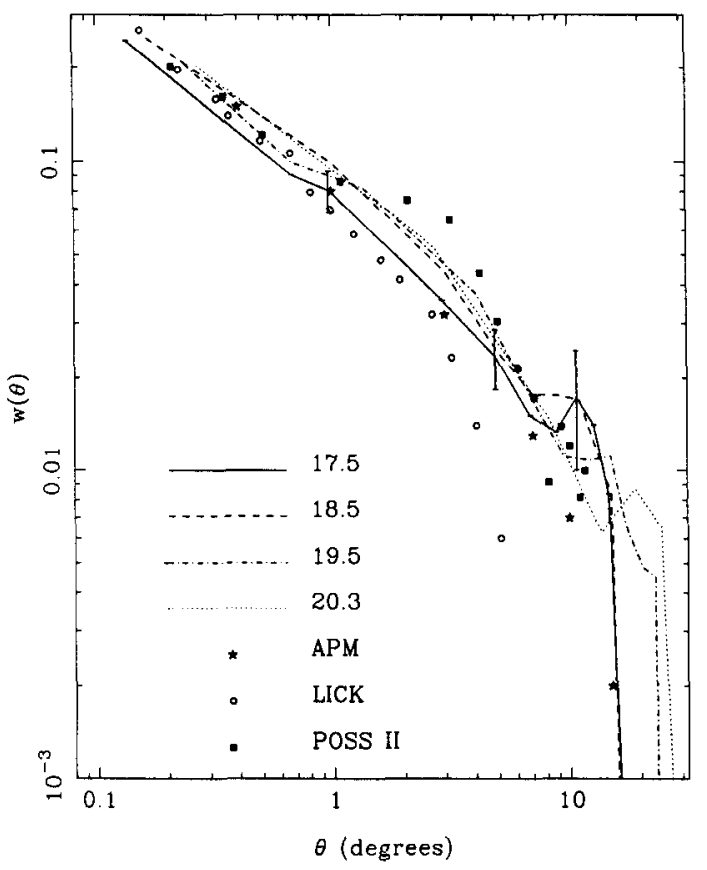

Figure 3. The $w(\theta)$ of the EDSGC at 4 magnitude limits. Also shown are the results from the Lick, APM and POSS II surveys. The digitised results all agree on the amplitude of $w(\theta)$. The $1 \sigma$ error bars, estimated empirically from the data, are indicated.

$10^{\circ}$, corresponding to $\simeq 30 h^{-1} \mathrm{Mpc}$. This excess clustering is above that of the Lick survey which has gone negative at $6^{\circ}$. This excess power is stable to the inclusion of all known systematics in the surveys (e.g. Nichol \& Collins 1993). A demonstration of this is presented in Figure 3, which shows that the EDSGC $w(\theta)$ at different magnitude limits all show the same excess power as each other and the APM correlation function.

Theoretical predictions for $w(\theta)$ are obtained directly from N-body simulations which trace the growth of structure from the initial density fluctuation spectrum. A comparison with theory demonstrates that these results do not support the canonical cold dark matter (CDM) theory of galaxy formation (e.g. Efstathiou et al. 1992b).

\section{The Spatial Correlation Function of Rich Clusters}

Clusters of galaxies are themselves clustered on scales $10-100 h^{-1} \mathrm{Mpc}$. As discussed in section 2, the Abell catalogue has been the most widely used source of clusters and the bench-mark for cluster correlation studies is the work of Bahcall \& Soneira (1983). If correct, their correlation length $\left(r_{0} \simeq 25 h^{-1} \mathrm{Mpc}\right)$ 
indicates that clusters are some 15 times more clustered than galaxies on the same spatial scale. More recently, various authors have confirmed a high amplitude for $r_{0}$ using more extensive redshift surveys of Abell clusters (Postman et al. 1986, 1992). As mentioned in section 2, some doubt has also been cast on these results due to the quality of the Abell catalogue. In addition to this, clusters selected from two-dimensional data are prone to projection effects. This happens when galaxies along the line-of-sight to a cluster get assigned as members of that cluster, thereby spuriously enhancing the richness. By modelling the Abell selection, Lucey (1983) suggests that $15 \%-20 \%$ of rich clusters in the Abell catalogue have a true membership that is less than half the observed membership. Sutherland (1988), Sutherland \& Efstathiou (1991) and Efstathiou et al. (1992a) argue that $r_{0}$ determinations using the Abell catalogue may well be spuriously enhanced by a factor of two.

In order to study $\xi_{c c}$, a redshift survey of the 100 richest systems in the EDSGC was carried out. The clusters were selected automatically using a surface density finding algorithm (Lumsden et al. 1992). Such automated selection removes the subjective biases inherent in the visually compiled Abell catalogue. There are three further aspects to our strategy which were specifically designed to reduce the problem of projection effects. First, the sample was corrected by deblending clusters which had overlapping radii. Galaxies in the overlap region were assigned to the appropriate cluster based on a Gaussian fit to the cluster density profiles. Secondly, in selecting the sample a projected cluster radius corresponding to $r_{A}=1.0 h^{-1} \mathrm{Mpc}$ was used in place of the $r_{A}=1.5 h^{-1} \mathrm{Mpc}$ value adopted by Abell. This reduced the number of cluster blends from $30 \%$ to about $8 \%$. Finally, the strategy behind the redshift survey was to measure the redshift of $\simeq 10$ galaxies towards the core of each cluster. The majority of existing cluster redshift surveys are based on only one or two galaxy redshift measurements per cluster. For example, the two largest cluster redshift surveys to date, the APM survey (Dalton et al. 1992) and by Postman et al. (1992) contain significant fractions of single or double cluster redshift measurements. The redshift of a cluster was defined in an objective manner following a similar prescription to that described by Struble \& Rood (1991).

Figure 4 shows the EDSGC cluster correlation function along with those of the Abell samples and the APM survey (Dalton et al. 1992). A least-squares fit to the EDSGC data gives $r_{0}=16.4 \pm 4.0 h^{-1} \mathrm{Mpc}$. Both the EDSGC and APM results are systematically below the Bahcall \& Soneira (1983) correlation function. One possibility for the difference between the Abell samples and the digitised data is that the digitised surveys pick out more compact clusters which have an intrinsically lower clustering amplitude (e.g. Bahcall \& West 1992). However, this argument depends on there being a correlation between $r_{0}$ and cluster richness, something which has only been established for Abell clusters (e.g. Efstathiou et al. 1992, Croft \& Efstathiou 1994). An alternative explanation for the difference is simply that the Abell sample is contaminated by projection effects which enhance the clustering along the line-of-sight. Figure 5 provides firm evidence for this effect. This shows the contours of $\xi_{c c}$ as a function of the radial (redshift) direction and the transverse direction. Figure $5 \mathrm{a}$ is the result obtained for the EDSGC clusters and Figure $5 \mathrm{~b}$ is the corresponding diagram for the Postman et al. (1992) redshift survey of $R \geq 1$ Abell clusters. On scales close to $r_{0}$ the contours of $\xi_{c c}\left(r_{z}, r_{p}\right)$ in Figure $5 \mathrm{~b}$ are elongated in the 


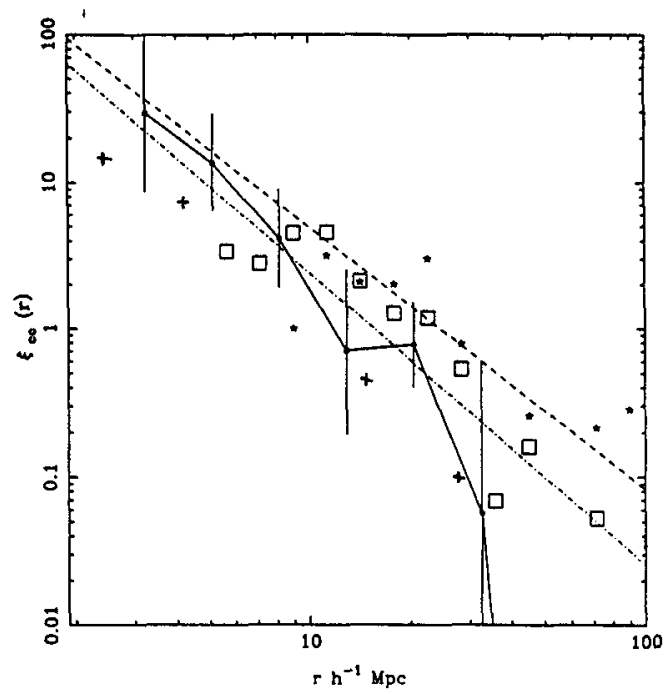

Figure 4. The $\xi_{c c}$ for the EDSGC clusters (•), along with the results for the richness class $R \geq 0(\square)$ and $R \geq 1(\star)$ Abell samples, and the APM catalogue $(+)$. The dot-dashed line represents the best fit to $\xi_{c c}(r)$ and the single-dashed line corresponds to the Bahcall \& Soneira $r_{0}=25 h^{-1} \mathrm{Mpc}$ correlation function. The bootstrap error bars are shown.

ratio of about 3 to 1 . In Figure $5 \mathrm{a}$ these elongations are reduced to about 2 to 1 . This strongly suggests that the anisotropy in the Abell sample is due to projection effects and is not the result of large cluster peculiar velocities or real line-of-sight clustering, as suggested by some authors (Bahcall et al. 1986, Jing et al. 1992). Using our data, we can place a firm upper limit of $1000 \mathrm{~km} \mathrm{~s}^{-1}$ on the peculiar velocity of clusters (Nichol et al. 1992). In the context of cosmological models, the data here are marginally consistent with standard CDM, although variants on the standard model do provide a better fit to the data (Mann et al. 1993, Croft \& Efstathiou 1994).

As a slight aside to the main discussion, I would like to point out that selecting clusters from photographic plates is not the optimum way to do this kind of experiment, whether the catalogues be Abell, EDSGC or APM. In all these cases clusters are selected by identifying galaxy surface density enhancements on plates, subject to a variable background. The cluster samples generated in this way are not unique. It is possible to change the final cluster selection significantly by slightly modifying one of the selection parameters. By contrast, selecting clusters in the $\mathrm{X}$-ray overcomes these difficulties. Figure $5 \mathrm{c}$ shows the anisotropy plot for an X-ray flux limited sample of 128 galaxy clusters selected from the ROSAT all-sky survey in a $3000 \mathrm{deg}^{2}$ region of the southern sky (Romer et al. 1994). The anisotropies seen in Figures $5 \mathrm{a}$ and $5 \mathrm{~b}$ have now completely disappeared. The correlation length for this sample is $r_{0}=13.7 \pm 2.3 h^{-1} \mathrm{Mpc}$, confirming the low values reported by the digitised surveys. 

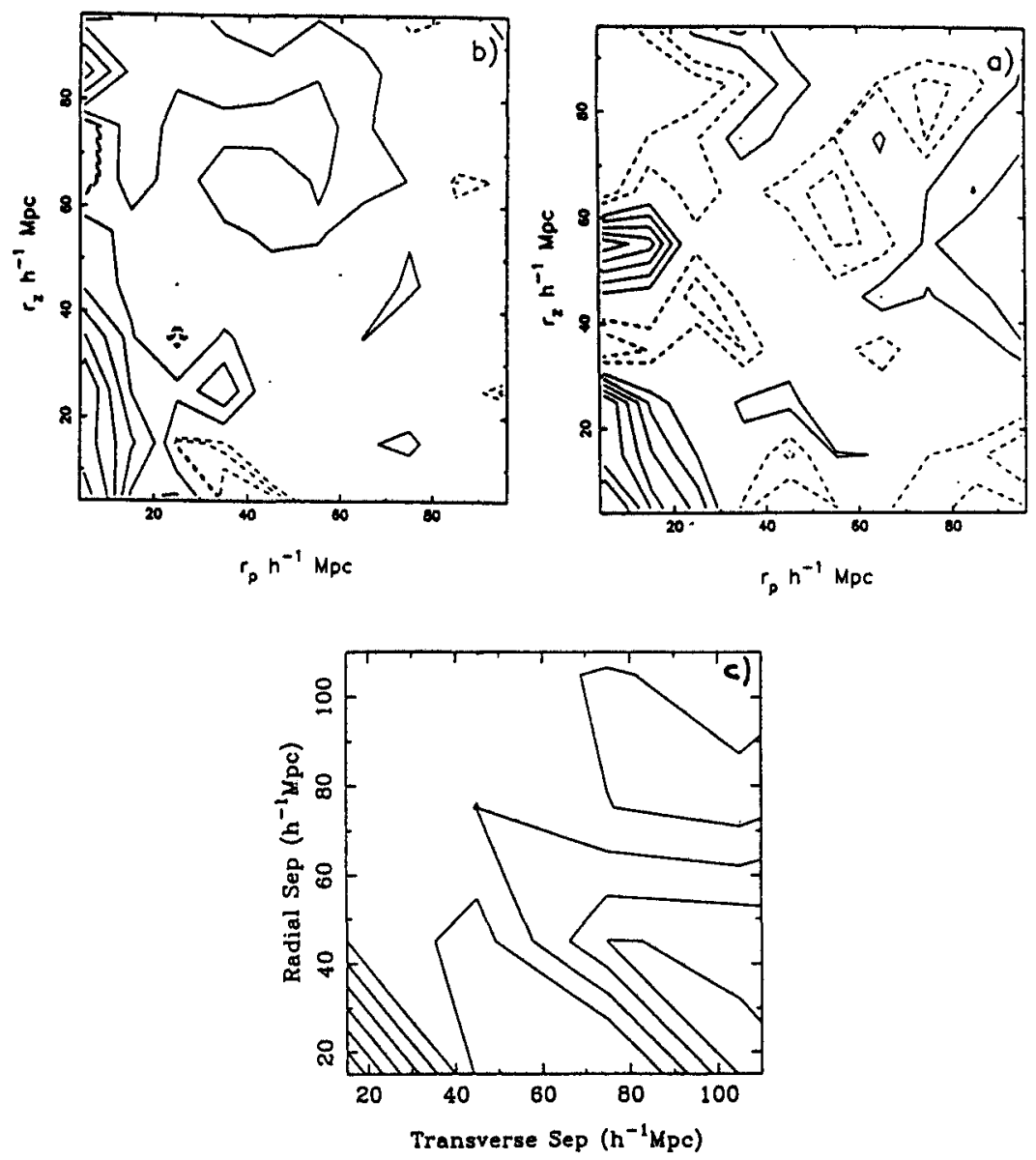

Figure 5. Contour diagrams of $\xi\left(r_{z}, r_{p}\right)$ for, (a) the EDSGC clusters, (b) the $R \geq 1$ Abell sample of Postman et al. (1992), (c) the ROSAT flux limited sample. Contour levels range from 12 to -0.5 . 

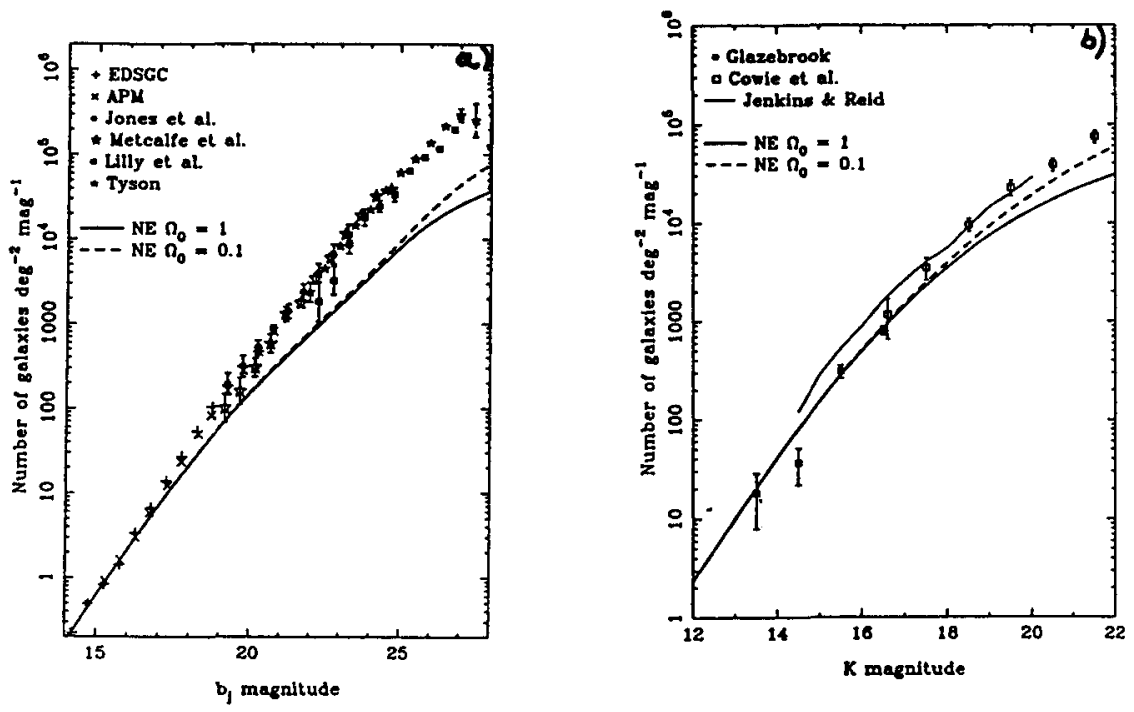

Figure 6. The differential galaxy number counts for, (a) CCD surveys and $4 \mathrm{~m}$ plates plus the EDSGC and APM photographic data, (b) near-IR K-band data. No-evolution models in two cosmologies are also shown.

\section{Galaxy Number Counts}

I would just like to say a few words on the topic of galaxy number counts. Only since the completion of the EDSGC and APM surveys have the number counts been known to high precision at bright magnitudes (see Figure 6a). Previously, no-evolution models of galaxy formation had been normalized to the data at about $b_{j} \simeq 18$, where there was enough signal to noise to determine the slope accurately. As a consequence of this, galaxy evolution did not appear to begin until $b_{j} \simeq 19-20$ (e.g. Ellis 1987). However, the EDSGC and APM surveys indicate a slope $\simeq 0.6$ in the counts brighter than $b_{j} \simeq 20$ (Heydon-Dumbleton et al. 1989, Maddox et al. 1990). The natural interpretation of this result is that galaxies are evolving at magnitudes $b_{j} \simeq 18.5$, corresponding to $z \simeq 0.1$, although the possibility of a large hole in the galaxy distribution cannot be ruled out (Shanks 1990, Maddox et al. 1990). Interestingly, the situation in the near-infrared is very different. Here, the $\mathrm{K}$ band counts do not show such strong evolution (see Figure $6 \mathrm{~b}$ ). A detailed comparison of the counts in the optical and $\mathrm{K}$ band, along with constraints from the $N(z)$, suggests that the best model to explain this data is to invoke rapid galaxy merging that induces bursts of star-formation (e.g. Glazebrook et al. 1994). 


\section{Future Prospects}

I would like make a few comments on the future use of Schmidt telescopes for these studies. First, a request to Neill Reid, "please finish off POSS II". As John Huchra made clear to me a while ago, the comparisons between the digitised surveys and both the Lick and Abell catalogues, are also comparisons between the northern and southern hemispheres. We have to know if the clustering properties are the same on the largest angular scales. Secondly, my belief is that only by imaging very deep will substantial progress be made in understanding galaxy clustering and the link with cosmological models. The work on galaxy number counts indicates that galaxy evolution and the evolution of large-scale structure are closely coupled phenomena and that galaxy evolution takes place over recent look-back times $z \simeq 0.2-0.4$. At these redshifts the merging model reduces the size of typical $L^{\star}$ galaxies to $\simeq 2$ arcsec. Future redshift surveys probing to depths $z \simeq 0.4$ will need to be based on good quality high spatial resolution CCD imaging, reaching $b_{j} \simeq 25$. Finally, statistical studies of galaxy clustering at progressively fainter depths puts a progressively greater demand on the required photometric accuracy. This accuracy will probably be limited by galactic extinction and future CCD surveys examining faint clustering will be confined to about $\pi$ sr in each hemisphere.

\section{Remember This Stuff}

- Digitised catalogues have had enormous impact on our understanding of the distribution of galaxies and clusters.

- There is too much clustering for $\Omega=1 \mathrm{CDM}$.

- The distribution of galaxy clusters is isotropic.

- Photographic number counts suggest evolution at low redshift $z \simeq 0: 1$.

- Future clustering studies will rely on deep high resolution CCD imaging surveys.

\section{Acknowledgments}

As with many other programmes, the clustering work presented here relied heavily on excellent Ph.D. students. I warmly thank my two ex-students, Bob Nichol and Kath Romer for their efforts.

\section{References}

Aaronson M., Bothun G., Mould H., Huchra J., Schommer R. \& Cornell M., 1986, ApJ, 302, 536

Abell G. O., 1958, ApJS, 3, 211

Abell G. O., Corwin H. G. \& Olowin R. P., 1989, ApJS, 70, 1

Bahcall N. A. \& Soneira R. M., 1983, ApJ, 311, 15 
Bahcall N. A., Soneira R. M. \& Burgett W. S., 1986, ApJ, 311, 15

Bahcall N. A. \& West M., 1992, ApJ, 392, 419

Collins C. A., Nichol R. C. \& Lumsden S. L., 1992, MNRAS, 254, 295

Cowie L. L., Gardner J. P., Lilly S. J. \& McLean I. S., 1990, ApJ360, L1

Cowie L. L., 1991, in Observational Tests of Inflation, T. Shanks, ed., (Kluwer, Dordrecht), p. 257

Croft R. A. C. \& Efstathiou G., 1994, MNRAS, in press

Dalton G. B., Efstathiou G., Maddox S. J. \& Sutherland W., 1992, ApJ, 390, L1

Efstathiou G., 1990, in Physics of the Early Universe, J. A. Peacock, A. F. Heavens \& A. T. Davies, eds, Proc. of the Thirty Sixth Scottish Universities Summer School in Physics, Edinburgh, p. 361

Efstathiou G., Dalton G. B., Sutherland W. J. \& Maddox S. J.,1992, MNRAS, 257,125

Efstathiou G., Bond J. R. \& White S. D. M., 1992, MNRAS, 258, 1p

Ellis R. S., 1987, in Observational Cosmology, Proc. IAU Symp. 124, A. Hewitt, G. Burbidge \& L.Z. Fang, eds, (Dordrecht, Reidel), p. 367

Fong R., Hale-Sutton D. \& Shanks T., 1992, MNRAS, 257, 650

Geller M. J., de Lapparent V. \& Kurtz M. J., 1984, ApJ, 287, L55

Glazebrook K., Peacock J. A., Collins C. A. \& Miller L., 1994, MNRAS, 266, 63

Groth E. J. \& Peebles P. J. E., 1977, ApJ, 217, 385

Groth E. J. \& Peebles P. J. E., 1986a, ApJ, 310, 499

Groth E. J. \& Peebles P. J. E., 1986b, ApJ, 310, 507

Heydon-Dumbleton N. H., Collins C. A. \& MacGillivray H. T., 1989, MNRAS, 238,379

Jenkins C. R. \& Reid I. N., 1991, AJ, 101, 1595

Jing Y-P., Plionis M. \& Valdarnini R., 1992, ApJ, 389, 499

Jones L. R., Fong R., Shanks T., Ellis R. S. \& Peterson B. A., 1991, MNRAS, 249,481

Lilly S. J., Cowie L. L. \& Gardner J. P., 1991, ApJ, 369, 79

Lucey J. R., 1983, MNRAS, 204, 33

Lucey J. R. \& Carter D., 1988, MNRAS, 235, 1177

Lumsden S. L., Nichol R. C., Collins C. A. \& Guzzo L., 1992, MNRAS, 258, 1.

Metcalfe N., Shanks T., Fong R. \& Jones L. R., 1991, MNRAS, 249, 498

Maddox S. J., Efstathiou G., Sutherland W. J. \& Loveday J., 1990, MNRAS, $242,43 p$

Mann R. G., Heavens A. F. \& Peacock J. A., 1993, MNRAS, 263, 798

Mathis J. S., 1990, ARA\&A, 28, 37

Nichol R. C., Collins C. A., Guzzo L. \& Lumsden S. L., 1992, MNRAS, 255, 21p

Nichol R. C. \& Collins C. A., 1993, MNRAS, 265, 867

Picard A., 1991, ApJ, 368, L7

Postman M., Geller M. J. \& Huchra J. P., 1986, AJ, 91, 1267 
Postman M., Huchra J. P. \& Geller M. J., 1992, ApJ, 384, 404

Romer A. K., Collins C. A., Bohringer H., Cruddace R. G., Ebeling H., MacGillivray

H. T. \& Voges W., 1994, Nature, submitted

Shane C. D. \& Wirtanen C. A., 1967, Publs Lick Obs., 22, Part 1

Shanks T.S., 1990, in The Galactic and Extragalactic Background Radiation, S. Bowyer \& C. Leinert, eds, p. 269

Stark A. A., Gammie C. F., Wilson R. W., Balley J., Linke R. A., Heiles C. \& Hurwity M., 1992, ApJS, 79, 77

Struble M. F. \& Rood H. J., 1991, ApJ, 374, 395

Sutherland W., 1988, MNRAS, 234, 395

Sutherland W. \& Efstathiou G., 1991, MNRAS, 248, 159

Tyson J. A., 1988, AJ, 96, 1

\section{Discussion}

Gilmore: You have noted the complementary information from comparing $b_{j}$ and $\mathrm{K}$ counts at relatively bright magnitudes. Would you comment on the desirability of adding $R$ colour data, to your, or the APM surveys, compared to deeper clustering data.

Collins: I think that the fact that the number count evolution is wavelength dependent, argues strongly for studying both clustering and evolution as a function of colour. This is done most effectively using multi-colour CCD surveys in order to reach the necessary depths to study clustering at $z=0.2-0.4$.

Peterson: The change in slope at $\sim 19$ th magnitude in the $b_{j}$ number counts is also seen in the K-band counts, indicating that this is most likely caused by a "local" galaxy under-density, rather than being due to galaxy luminosity evolution between 15th and 19th magnitude.

Collins: This is possible, but we will have to wait for accurate digitized data from POSS II to distinguish between these possibilities.

Padmanabhan: I agree that deeper surveys would be of relevance in studying galaxy evolution. However, do you think this would help us discriminate between different models of structure formation? I would have thought that the astrophysical uncertainty in model-building will make this a difficult task.

Collins: These observations should provide the necessary data which will be the driving force behind future theoretical work in this area, however complicated these may turn out to be. 